\title{
Paediatric endophthalmitis: a UK retrospective study
}

\author{
S. Parvizi ${ }^{1,2} \cdot$ M. Papadopoulos ${ }^{1} \cdot$ V. Panteli ${ }^{2} \cdot$ J. Brookes $^{1,2} \cdot$ J. Soothill ${ }^{2} \cdot$ J. Bloom $^{1} \cdot$ G. G. W. Adams ${ }^{1}$. \\ M. Theodorou ${ }^{1,3}$
}

Received: 1 June 2018 / Revised: 12 March 2019 / Accepted: 18 June 2019 / Published online: 12 August 2019

(c) The Author(s), under exclusive licence to The Royal College of Ophthalmologists 2019

\begin{abstract}
Introduction Paediatric endophthalmitis is a severe but rare complication of intraocular surgery, penetrating trauma and far less commonly extra-ocular surgery or endogenous origin. We set out to establish the incidence and risk factors of exogenous endophthalmitis in children, and to develop an evidence-based protocol that can be used for treatment of suspected exogenous endophthalmitis in children.

Methods Microbiology reports and operation numbers were obtained from two large tertiary referral hospitals sharing 24-h paediatric ophthalmology cover for the period January 2009-December 2016. All cases of aqueous and/or vitreous tap performed on children aged $\leq 18$ years were identified and case notes reviewed for complete information on each case.

Results Sixteen cases were eligible for inclusion as 'postoperative endophthalmitis': complete data was found on 13 cases. The incidence of postoperative endophthalmitis was $0.17 \%$ over 7 years.

The mean age of presentation was 5.5 years (range from 7 months to 16 years and 9 months), from 3 days- 78 months post operatively. In all, 11/13 had at least one glaucoma procedure. Microbiology results showed growth in 8/13. Most isolates were Gram-positive bacteria but Gram-negatives were also isolated and in one case Candida from a conjunctival swab. The antibiotic regime varied depending on age, organism identified and sensitivities. In all, 9/13 had hand movement or worse vision after treatment.

Conclusion Paediatric endophthalmitis may present to any paediatric/general ophthalmologist. It is a rare but devastating condition with poor visual prognosis, requiring prompt recognition and aggressive management. Previous glaucoma surgery is a long-term risk factor in our local paediatric population. Based on our study, an evidence-based protocol for management is proposed in order to improve outcomes.
\end{abstract}

\section{Introduction}

Paediatric endophthalmitis is a severe but rare complication of intraocular surgery and penetrating trauma. Very rarely it can complicate extra-ocular surgery, and a few cases are from an endogenous source. It may present to any paediatric or general ophthalmologist, with devastating visual consequences requiring prompt and aggressive management. There is considerable literature on adult endophthalmitis, but less published on exogenous endophthalmitis and its

\footnotetext{
S. Parvizi

sahar.parvizi@gmail.com

Moorfields Eye Hospital, London, UK

2 Great Ormond Street Hospital, London, UK

3 National Institute for Health Research Biomedical Research Centre at Moorfields Eye Hospital, London, UK
}

treatment in the paediatric age group [1-10]. More recently, online surveys of the American Association for Paediatric Ophthalmology and Strabismus members on cases of endophthalmitis following paediatric cataract surgery [11] as well as strabismus surgery [12] reported 22 and 27 cases, respectively.

As a result, to date, there are no well-established evidence-based guidelines for use in infants and children. Current standard clinical practice in the UK is to make appropriate adjustments to available adult protocols.

In adults, cataract surgery is a common intraocular procedure with approximately 390,000 operations performed in England each year [13]. In children, strabismus surgery is the most common surgical procedure. Non-traumatic paediatric intraocular surgery tends to be managed in a few specialist centres in the UK, due to the low incidence of around 250 cases per year of paediatric cataracts [14], and the need for specialist intra- and post-operative management, particularly in infants. Endophthalmitis has been 
reported following strabismus surgery but is thought to be rare, with case reports making up the majority of the published literature and a reported incidence of one in 24000 in the UK [15]. Scleral perforation is thought to be a prerequisite for the development of endophthalmitis following strabismus surgery, estimated to occur in $0.08 \%$ [15]. This has been greatly reduced following the introduction of spatulated needles allowing separation of the scleral lamellae, and the use of surgical techniques to avoid where possible, suturing in areas where the sclera is thinned.

Trauma and glaucoma procedures have been reported to be the commonest causes of exogenous endophthalmitis in children. The incidence of endophthalmitis after penetrating injury is up to $20 \%$, and may be even higher when the injury has occurred in a rural setting and/or presentation is delayed [5-9]. Bleb-related infections (blebitis and blebrelated endophthalmitis) are thought to occur more frequently in children compared to adults due to poor hygiene [16]. Bleb-related endophthalmitis (BRE) has been reported in up to $9 \%$ of paediatric mitomycin $\mathrm{C}$ trabeculectomies $[17,18]$. Endophthalmitis associated with glaucoma drainage devices (GDD) is a rare complication but a serious one reported with all currently available GDD. The exact incidence is not known [6-9, 19-22], but again it is more common in children, who have a five times higher rate of endophthalmitis compared to adults with Ahmed valve implants: $4.4 \%$ in children versus $0.9 \%$ in adults over a 9 year follow-up period [19]. Conjunctival erosion and tube exposure are significant risk factors for delayed postoperative intraocular infections allowing organisms intraocular access. The pathogens associated with GDD-related endophthalmitis tend to be similar to those associated with BRE such as Pseudomonas aeruginosa, Haemophilus influenzae and Streptococcus species [19, 22].

Despite the current literature, the presentation and etiology of paediatric endophthalmitis is still not well recognised amongst general and paediatric ophthalmologists and there is not, to date, an established protocol for managing these difficult cases in the UK. This study was designed to establish current practice and outcomes in two busy tertiary referral centres in the UK, with a view to developing a protocol which can be used in similar settings. A retrospective case notes review of all cases of endophthalmitis was performed to determine the risk factors, microbiology and management, with the aim of developing an evidencebased protocol for management that could potentially be employed nationally.

\section{Methods}

A retrospective case note review was performed. The review was approved by the local audit committee at
Moorfields Eye Hospital NHS Foundation Trust (reference: CA15/ONSP/10). Authors SP and VP collated the data on all patients younger than 18 years who presented to Moorfields Eye Hospital London and Great Ormond Street Hospital London over a 7-year period (January 2009-December 2016). The two tertiary referral hospitals are involved in joint 24-h paediatric ophthalmology cover within London and its surrounding areas.

The total number of paediatric intraocular and strabismus procedures was obtained using search terms and codes on the electronic theatre information system across both hospitals. All strabismus and intraocular surgical cases were included: glaucoma, cornea, cataract, vitreoretinal and posttraumatic repairs.

To accurately identify the endophthalmitis cases, microbiology reports were obtained of all cases of aqueous and/or vitreous taps performed on children aged $\leq 18$ years at both sites. Case notes were reviewed (paper and/or electronic) to collate complete information on each case, specifically information on demographics, past ocular history (including primary/secondary diagnoses, operation (s) performed and date(s), last documented visual acuities preceding the onset of exogenous endophthalmitis, date of admission with endophthalmitis, treatment received following diagnosis of endophthalmitis and last visual acuity documented in the clinical notes following treatment. Cases of confirmed endogenous endophthalmitis were excluded.

\section{Results}

\section{Incidence}

A total of 9507 cases of strabismus as well as intraocular procedures including lensectomies, penetrating keratoplasties, glaucoma procedures and post-traumatic surgical repairs were performed across the two sites over a 7-year period.

Microbiology reports of aqueous and/or vitreous samples were retrieved in 38 children. Twenty two were excluded for the following reasons: samples were taken for a nonendophthalmitis indication $(n=18)$; external hospital samples $(n=3)$ and a sample from a confirmed case of endogenous endophthalmitis $(n=1)$. In all, 16/38 (42.1\%) were eligible for inclusion in the analysis. We were unable to obtain complete medical and operative notes in three cases, so only 13 cases were included in the descriptive analysis. Overall, the incidence of exogenous endophthalmitis in our study was $0.17 \%$ over 7 years (16/9507), with all cases of infection expected to return to the original hospital in which surgery was carried out. 


\section{Patient characteristics}

Patient characteristics are summarized in Table 1. The mean age at presentation was 5.5 years (range from 7 months to 16 years 9 months). In all, 9/13 (69.2\%) were female. In all, $11 / 13(84.6 \%)$ children had glaucoma as their primary diagnosis; $1 / 13$ (7.7\%) had anterior segment dysgenesis; $1 / 13(7.7 \%)$ had trauma.

The children had a range of procedures. In all, 11/13 $(84.6 \%)$ had at least one glaucoma procedure including goniotomy, trabeculectomy and GDD surgery (Ahmed/ Baerveldt implants). In all, 1/13 (7.7\%) had a penetrating keratoplasty for anterior segment dysgenesis, and 1/13 (7.7\%) had a lensectomy and secondary intraocular lens implant following penetrating trauma.

The mean time from the last surgical procedure to presentation was 15 months (range from 3 days to 6 years 6 months). The earliest post-operative presentation was 3 days post lensectomy for a traumatic white cataract and primary repair for penetrating injury.

The GDDs were removed in eight cases with an Ahmed or Baerveldt implant in situ at the time of acute treatment for endophthalmitis, at a mean time of 25 days (range 0-60 days) after admission with infection. Only two of these cases had clearly documented tube exposure and conjunctival erosion.

Monocular visual acuities were recorded between 0.34 and $1.45 \log$ MAR in four children before endophthalmitis. The remaining nine children had binocular or unmeasurable visual acuities. The final documented visual acuity was poor in all, ranging at best from 1.0 LogMAR to no perception of light/phthisical eyes in 5/13 (38.4\%).

\section{Microbial growth and treatment}

All 13 children had an Examination Under Anaesthesia (EUA), aqueous and/or vitreous samples taken, and intraocular antimicrobials administered. The microbial samples, antimicrobial and corticosteroid regimes used in each patient are summarised in Table 2 .

Eight had positive microbiological samples shown in Fig. 1; five had no growth documented. In all, 7/8 grew Gram-positive bacteria (five only Gram-positive; two mixed Gram-positive and Gram-negative growth). In all, 1/8 grew candida on a conjunctival swab (with no growth from intraocular samples).

Intravitreal treatment was administered at the time of the EUA in all cases. In all, 10/13 (76.9\%) were given intravitreal vancomycin and amikacin (two also received intravitreal dexamethasone), 2/13 (15.4\%) were given intravitreal vancomycin alone, and 1/13 (7.7\%) was given intravitreal vancomycin and amphotericin due to the mechanism of injury (penetrating trauma with a pencil).
All children received systemic antibiotics, although the antibiotic regime varied as there was no standard paediatric protocol available at the time. 10/13 received a quinolone (ciprofloxacin in $9 / 10$, moxifloxacin in $1 / 10$ ), which was augmented with Gram-positive cover in $5 / 10$ and/or antifungals in $2 / 10$. Six children were given oral/intravenous antibiotic monotherapy and three children were given triple antibiotic therapy. 3/13 received oral prednisolone.

Topical antibacterial treatment was given to all children. In all, 12/13 (92.3\%) were given a topical quinolone either alone in $7 / 12(58.3 \%, 53.4 \%$ of total) or augmented with either neomycin/maxitrol in $1 / 12(8.3 \%, 7.7 \%$ of total), vancomycin in $1 / 12(8.3 \%, 7.7 \%$ of total), chloramphenicol in $3 / 12(25 \%, 23 \%$ of total). In all, $1 / 13$ $(7.7 \%)$ received chloramphenicol as the sole topical agent. In all, 2/13 (15.4\%) received topical antifungals (econazole). Only $7 / 13$ children $(53.8 \%)$ received adjunctive topical corticosteroids.

\section{Discussion}

In this retrospective case series in a busy 24-h walk-in casualty covering two tertiary referral services, only 16 cases of exogenous endophthalmitis were identified in children under 18 years over a period of 7 years. This incidence of $0.17 \%$ increased to $1.16 \%$ when considering glaucoma procedures alone. Antimicrobial, antifungal and anti-inflammatory treatment varied, thus highlighting the need for the development of an evidence-based paediatric protocol.

Our case series differed from previously published papers where trauma accounted for a greater proportion of cases of endophthalmitis-44-86\% [6, 23]. In our study, trauma accounted only for one case, with glaucoma accounting for the majority. This reflects the case mix in our tertiary paediatric referral centres. Our findings may also suggest a reduced rate of endophthalmitis post trauma due to the increasing knowledge and effective antibiotic cover intra- and post-operatively as well as the availability of emergency paediatric anaesthetic and ophthalmic surgical facilities and staff, allowing early surgical intervention of a child with a traumatic open globe.

Glaucoma surgery is a well-recognized risk factor for endophthalmitis. However, due to the segregation of subspecialist paediatric glaucoma services, it may not be readily anticipated by non-glaucoma and/or paediatric specialists. The incidence of endophthalmitis post glaucoma surgery in our series is much lower than previously reported [5-9, 19-22]. This is likely to be multifactorial, and may include the early recognition and management of risk factors; for example, aggressive treatment of infective conjunctivitis, suspected blebitis and exposed glaucoma 


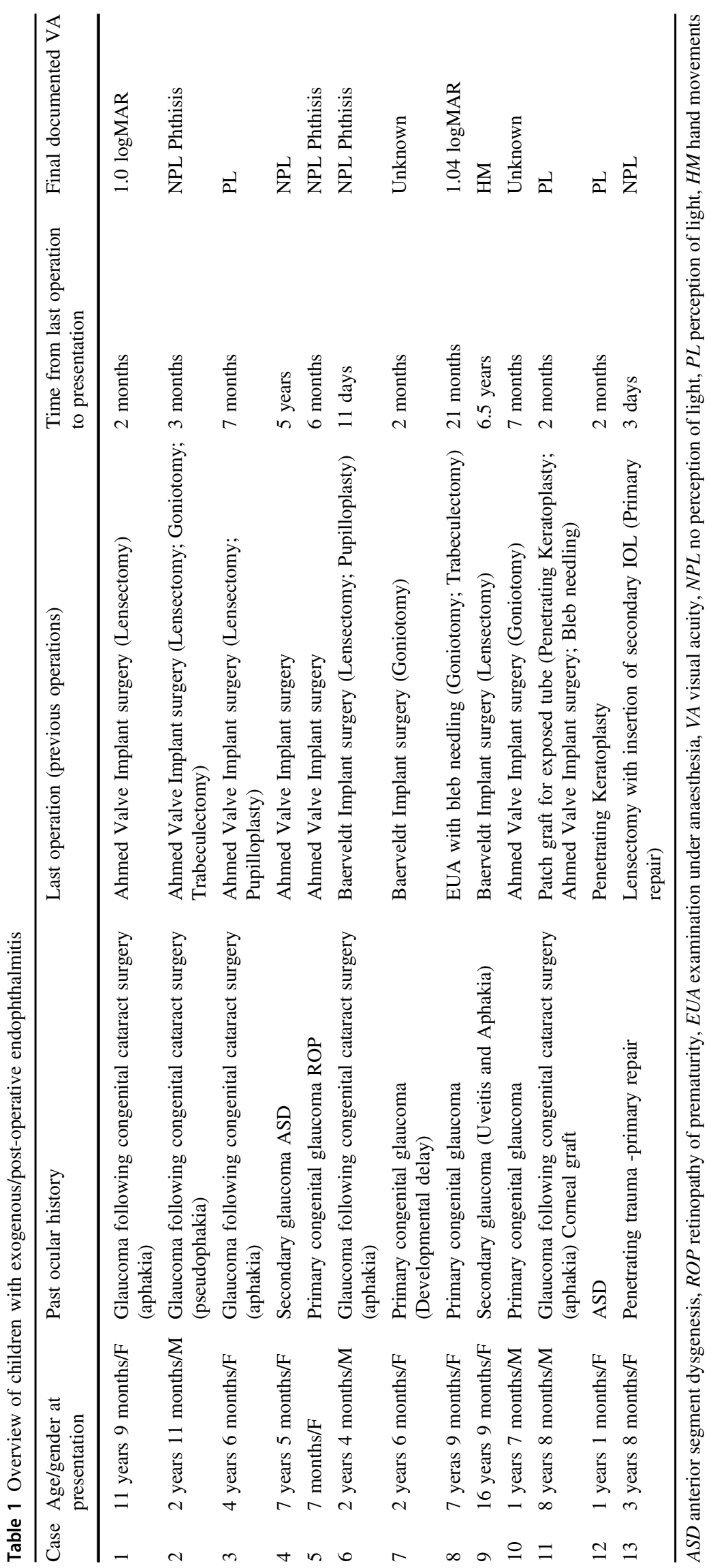




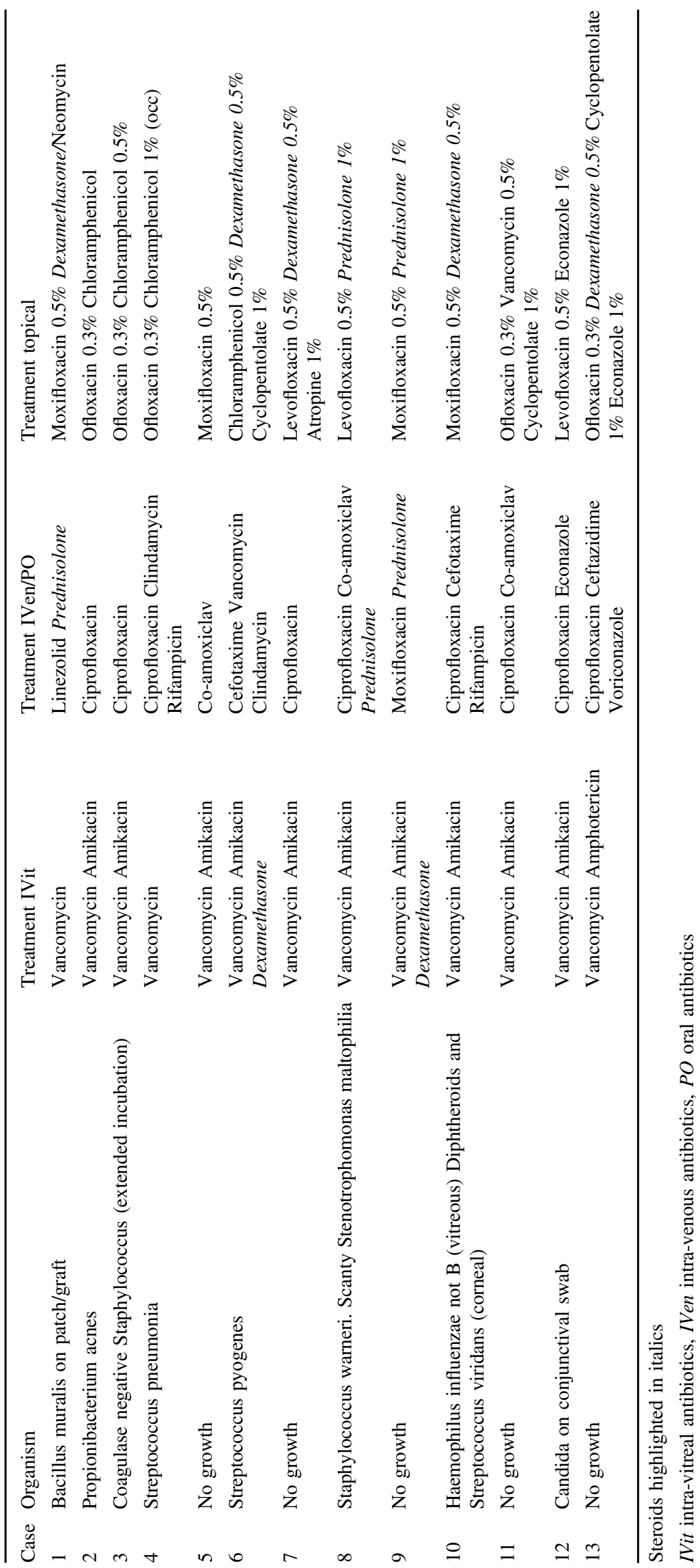




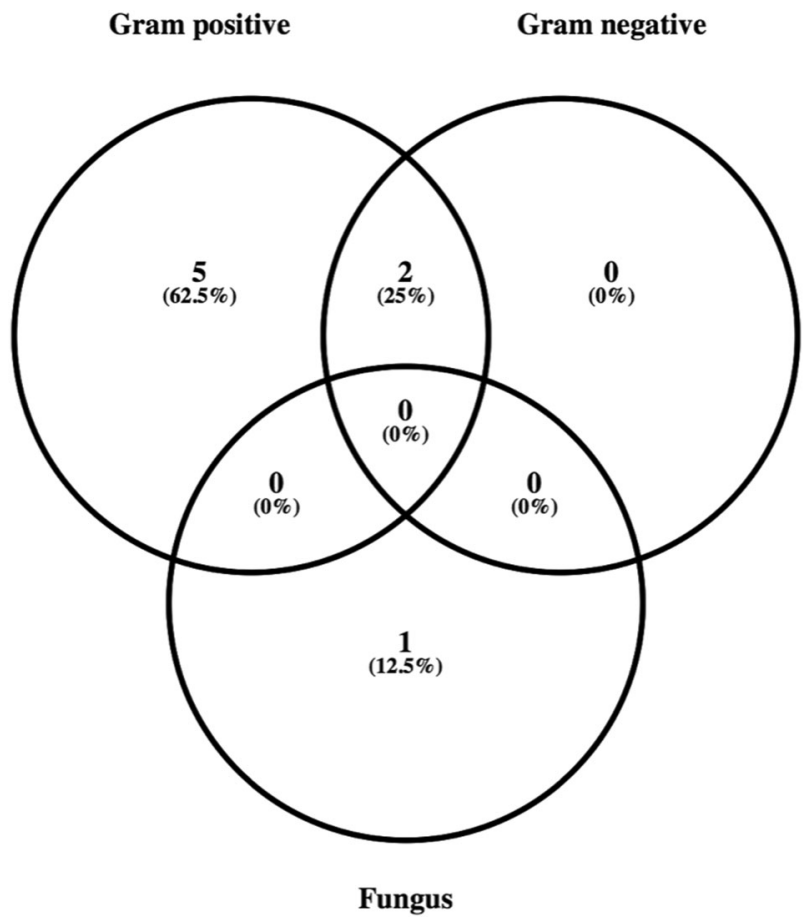

Fig. 1 Venn diagram summarizing organisms based on microscopy and Gram stain results in eight children. Five children showed no organisms on microscopy, or growth after prolonged period

tubes and/or plates, as well the improvement of surgical techniques.

In our case series, most endophthalmitis was associated with GDDs, which are commonly used as primary surgery for glaucoma associated with aphakia/pseudophakia, uveitis and anterior segment dysgenesis in our subspecialist services. Furthermore, recent modifications to the paediatric trabeculectomy technique and to the intraoperative application of mitomycin C (Moorfields Safer Surgery System) have reduced the incidence of thin avascular blebs and their associated complications [24, 25]. Ahmed valve implants, which featured strongly in our patient group, were the GDD of choice of a former surgeon in one of the centres.

As previously described, the presentation is often delayed, with children and adults commonly presenting months to years after the surgical procedure and so may be missed as being a relevant risk factor. This is particularly significant in children who are 'difficult to examine' as the delay in making the diagnosis may be very costly to their sight and even their eye. In our series, only two cases were documented prior to presentation as having thin, avascular blebs and so recognized as being at higher risk of developing endophthalmitis. The symptoms, signs and significance of bleb-related infection must be made clear to the parents who should be instructed to seek immediate attention. Long term visual prognosis depends on the extent of the infection, virulence of the organism and the timing of therapy. Bleb-related infection should be treated early and aggressively to reduce the risk of intraocular spread and so maximize visual function.

Delayed post-operative endophthalmitis has a different mechanism to the acute post-operative cases of endophthalmitis where the organism is thought to be introduced at the time of surgery. Endophthalmitis following initial GDD surgery usually occurs late due to: surgery associated with tube repositioning, capsulectomy, needling of the capsule and exposure of the device. Tube exposure is a major risk factor for endophthalmitis [22]. Although exposure can occur anywhere along the length of the tube or plate, it usually occurs over the tube at the limbus [19]. In a series of 60 paediatric eyes with Ahmed implants, the rate of tube exposure in children was high at $12 \%$ and endophthalmitis $3 \%$ [21]. This emphasizes the importance of inspecting the overlying conjunctiva for tube exposure whenever the child is examined and parents being warned should there be a thin layer of conjunctiva only over the tube.

The pathogenesis of bleb-related infection is believed to involve either direct spread through a ruptured, leaking bleb or transconjunctival migration of bacteria from the ocular surface into the eye through a very thin-walled filtering bleb. This may be aided by the toxins produced by some organisms, such as Streptococcus spp and Moraxella spp, although the mechanism of penetration through a nonleaking bleb has not been established.

Studies from the adult literature on bleb-related infection report a different, more virulent spectrum of organisms, such as Streptococcal species, Haemophilus influenza and Pseudomonas aeruginosa [26] compared to those causing acute post-surgical endophthalmitis, which are usually Gram-positive organisms introduced at the time of surgery [27]. The pathogens associated with GDD-related endophthalmitis tend to be similar $[19,22]$. In our case series, all positive cultures were Gram-positive (with 1/7 showing mixed growth), in keeping with previous reports. Bleb- and GDD-related endophthalmitis with Streptococci and Gramnegative species is usually associated with a poor visual prognosis. Streptococcal exotoxins may be retinotoxic, stimulating increased intraocular inflammation. Streptococcus was predominant in our cases series, with a poor visual prognosis where visual acuities ranged at best from 1.0 LogMAR to phthisical eyes/no perception of light. However, in children it is not only the virulence of the organism and exaggerated inflammatory response, but the inevitable delay in diagnosis and definitive treatment (requiring a general anaesthetic) which are also likely to contribute to the poor outcome. Candida was cultured from conjunctival swabs in one case, but organisms isolated from external and intraocular specimens may not necessarily be the same.

In most cases, intraocular antibiotics were consistent and similar to those used in adult protocols. Intravitreal 
vancomycin and a third-generation cephalosporin, ceftazidime, provide the definitive treatment and should be given with minimum delay. This choice of antibiotic is a move away from the previously used amikacin and gentamicin for Gram-negative cover due to the reported risk of retinal infarction [28]. Antibiotics should be amended based on preliminary microbiology results in conjunction with the clinical response. Many UK laboratories offer polymerase chain reaction (PCR) testing, which allows earlier tailored microbiological treatment, often within $24 \mathrm{~h}$.

However, due to the inevitable delay in arranging intraocular injections in children, the prompt administration of systemic antibiotics to ensure suitable cover of Streptococci with good intraocular penetration is more important in the paediatric age group, as opposed to the older children and adults who can be treated with intravitreals under local anaesthesia. Systemic ciprofloxacin (moxifloxacin above 12 years) provides good Gram-negative and some Grampositive cover, achieving bactericidal levels even in uninflamed eyes [29]. However, the Gram-positive cover may not always be adequate, particularly for streptococcal infections. We propose using a combination of both clindamycin and rifampicin for additional systemic Grampositive cover: clindamycin covers Staphylococcus spp., Streptococcus spp. and most anaerobes, and there is some evidence that it penetrates the eye. Rifampicin has wellestablished intra-ocular penetration [30]. It is very active against many Gram-positive bacteria although resistance is common with monotherapy, and its use should enhance the Gram-positive cover when used as triple therapy.

Although it is unclear from the literature whether the GDD should be removed when infected, in eight out of nine cases in our series it was removed. Removal of the implant in the acute phase is challenging because of excessive bleeding that occurs in an inflamed eye but is sometimes necessary if the infection does not settle despite antibiotics. There are reports of successful treatment with the GDD in situ but in most cases the implants are removed as the GDD is seeded with the pathogen and the implant as a foreign body is difficult to sterilize $[19,21]$.

The role of corticosteroids remains controversial. However, due to the marked inflammatory response in children, which may be exaggerated in the presence of microbial-associated exotoxins, the administration of adjunctive corticosteroids may play an important role in minimizing collateral inflammatory damage. Where there are no contraindications such as suspected fungal infection, intravitreal dexamethasone may be given intravitreally at the time of primary EUA (with care to monitor the post operative intraocular pressure), with a view to commencing oral prednisolone $24 \mathrm{~h}$ later.

We propose a pathway, which may be used in cases of paediatric exogenous endophthalmitis (Fig. 2).
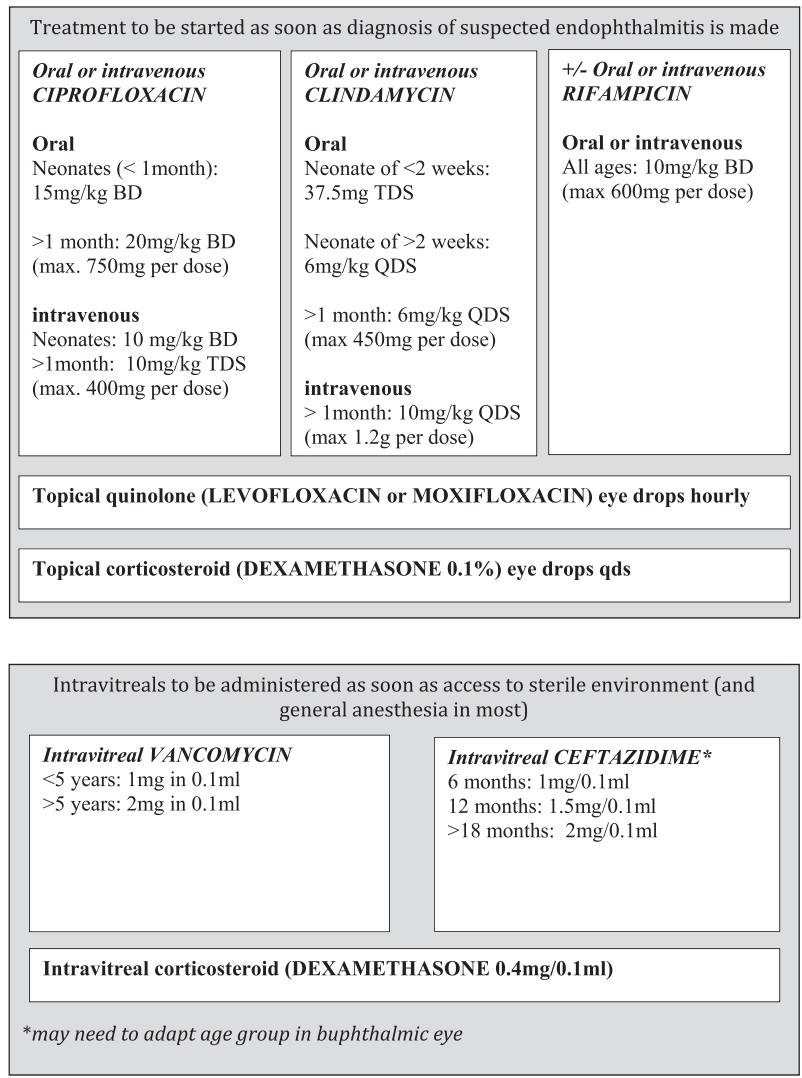

Fig. 2 Proposed treatment based on study and multidisciplinary agreement

\section{Limitations of the study}

Our study had the limitations of an uncommon presentation and retrospective data collection. Data was not available in $3 / 16$ cases, and the required data (e.g. frequency of application of medication or dosage, and findings at time of intravitreal injection) was not always documented clearly. The data is from two large inner city tertiary referral centres and may not necessarily be extrapolated to other units. Due to the acute/subacute presentation of these children to the original treating hospital, emergency treatment was provided by the on-call paediatric ophthalmologist at the time based on available adult protocols as well as advice from the on-call microbiologist. As a result, surgical technique and treatment regimens differed.

However, our study provides valuable information on a rare but sight-threatening complication of intraocular surgery, which can present to any ophthalmologist in or out of normal working hours. In contrast to previous literature, glaucoma surgery is the most common risk factor in our cases series, and may present months to years following surgery, often caused by virulent Streptococcal species. Increasing awareness will allow early recognition and prompt evidence-based management, with the aim of 
improving the poor visual outcomes in these children who have a lifelong risk of infection.

\section{Summary}

\section{What was known before}

- Paediatric endophthalmitis is a severe but rare complication of intraocular surgery, penetrating trauma, and far less commonly extra-ocular surgery or endogenous origin.

- Knowledge around the risk factors of post-operative endophthalmitis in children and how it is managed are limited.

\section{What this study adds}

- In this retrospective observational study, including 13 children under the age of 18 , the incidence of postoperative endophthalmitis was $0.17 \%$ over 7 years with previous glaucoma surgery being a major risk factor for developing this disease. There was no prior consensus on how these cases should be managed.

- A dedicated protocol for managing children with endophthalmitis is needed in all hospitals performing intraocular surgery on this age group, or accepting emergency cases of possible infection, and families should be well informed.

\section{Compliance with ethical standards}

Conflict of interest MT is supported by the National Institute for Health Research (NIHR) Biomedical Research Centre based at Moorfields Eye Hospital NHS Foundation Trust. The views expressed are those of the author(s) and not necessarily those of the NHS or the NIHR. The funding organizations had no role in the design or in conducting this research. The remaning authors declare that have no conflict of interest.

Publisher's note: Springer Nature remains neutral with regard to jurisdictional claims in published maps and institutional affiliations.

\section{References}

1. Clavell C, Athwal L, Zarbin MA, Langer PD, Bhagat N. Pediatric infectious endophthalmitis: a case series. J Pedia Ophthalmol Strabismus. 2018;55:69-70.

2. Alfaro DV, Roth DB, Laughlin RM, Goyal M, Liggett PE. Paediatric post-traumatic endophthalmitis. $\mathrm{Br} \mathrm{J}$ Ophthalmol. 1995;79:888-91.

3. Agarkar S, Desai R, Jambulingam M, Sumeer SH, Raman R. Incidence, management, and visual outcomes in pediatric endophthalmitis following cataract surgery by a single surgeon. J Aapos. 2016;20:415-8.

4. Wu H, Ding $X$, Zhang $M, X u$ G. Pediatric posttraumatic endophthalmitis. Graefes Arch Clin Exp Ophthalmol. 2016; 254:1919-22.

5. Junejo SA, Ahmed M, Alam M. Endophthalmitis in paediatric penetrating ocular injuries in Hyderabad. J Pak Med Assoc. 2010;60:532-5.

6. Thordsen JE, Harris L, Hubbard GB 3rd. Pediatric endophthalmitis. A 10-year consecutive series. Retina. 2008;28(3 Suppl):S3-7.

7. Khan S, Athwal L, Zarbin M, Bhagat N. Pediatric infectious endophthalmitis: a review. J Pedia Ophthalmol Strabismus. 2014;51:140-53.

8. Zhang M, Xu GZ, Jiang R, et al. Pediatric infectious endophthalmitis: a 271-case retrospective study at a single center in China. Chin Med J (Engl). 2016;129:2936-43.

9. Ranganath A, Hashim. A Late-onset endophthalmitis secondary to exposed glaucoma tube implant in a rare case of paediatric glaucoma. 2011. Case Rep Ophthalmol Med. https://doi.org/10.1155/ 2011/183647.

10. Recchia FM, Baumal CR, Sivalingam A, Kleiner R, Duker JS, Vrabec TR. Endophthalmitis after pediatric strabismus surgery. Arch Ophthalmol. 2000;118:939-44.

11. Gharaibeh AM, Mezer E, Ospina LH, Wygnanski-Jaffe T. Endophthalmitis following pediatric cataract surgery: an International Pediatric Ophthalmology and Strabismus Council Global Perspective. J Pedia Ophthalmol Strabismus. 2018;55:23-29.

12. Zloto O, Mezer E, Ospina L, Stankovic B, Wygnanski-Jaffe T. Endophthalmitis following strabismus surgery: IPOSC Global Study. Curr Eye Res. 2017;42:1719-24.

13. Donachie PHJ, Sparrow JM. National Ophthalmology Database Audit Year 2 Annual Report 2017. Royal College of Ophthalmologists. Accessed 30th Nov 2017. https://www.rcophth.ac.uk/ standards-publications-research/annual-reports-2.

14. Rahi JS, Dezateaux C. Measuring and interpreting the incidence of congenital ocular anomalies: lessons from a National Study of Congenital Cataract in the UK. IOVS. 2001;42:1444-8.

15. Bradbury JA. What information can we give to the patient about the risks of strabismus surgery. Eye. 2015;29:252-7.

16. Golde KT, Gardiner MF. Bacterial conjunctivitis in children: a current review of pathogens and treatment. Int Ophthalmol Clin. 2011;51:85-92.

17. Beck AD, Wilson WR, Lynch MG, Lynn MJ, Noe R. Trabeculectomy with adjunctive mitomycin $\mathrm{C}$ in pediatric glaucoma. Am $\mathrm{J}$ Ophthalmol. 1998;126:648-57.

18. Sidoti PA, Belmonte SJ, Liebmann JM, Ritch R. Trabeculectomy with mitomycin-C in the treatment of pediatric glaucomas. Ophthalmology. 2000;107:422-9.

19. Gedde SJ, Scott IU, Tabandeh H, et al. Late endophthalmitis associated with glaucoma drainage implants. Ophthalmology. 2001;108:1323-7.

20. Krishna R, Godfrey DG, Budenz DL, et al. Intermediate-term outcomes of 350-mm(2) Baerveldt glaucoma implants. Ophthalmology. 2001;108:621-6.

21. Morad Y, Donaldson CE, Kim YM, Abdolell M, Levin AV. The Ahmed drainage implant in the treatment of pediatric glaucoma. Am J Ophthalmol. 2003;135:821-9.

22. Al-Torbak AA, Al-Shahwan S, Al-Jadaan I, Al-Hommadi A, Edward DP. Endophthalmitis associated with the Ahmed glaucoma valve implant. Br J Ophthalmol. 2005;89:454-8.

23. Weinstein GS, Mondino BJ, Weinberg RJ, Biglan AW. Endophthalmitis in a pediatric population. Ann Ophthalmol. 1979;11:935-43.

24. Wells AP, Cordeiro MF, Bunce C, Khaw PT. Cystic bleb formation and related complications in limbus-versus fornix-based 
conjunctival flaps in pediatric and young adult trabeculectomy with mitomycin C. Ophthalmology. 2003;110:2192-7.

25. Jayaram H, Scawn R, Pooley F, et al. Long-term outcomes of trabeculectomy augmented with mitomycin $\mathrm{C}$ undertaken within the first 2 years of life. Ophthalmology. 2015;122:2216-22.

26. Ciulla TA, Beck AD, Topping TM, Baker ASBlebitis. early endophthalmitis, and late endophthalmitis after glaucoma-filtering surgery. Ophthalmology. 1997;104:986-95.

27. Han DP, Wisniewski SR, Wilson LA, et al. Spectrum and susceptibilities of microbiologic isolates in the Endophthalmitis Vitrectomy Study. Am J Ophthalmol. 1996;122:1-17.
28. Campochiaro PA, Conway BP. Aminoglycoside toxicity-a survey of retinal specialists. Implic Ocul use Arch Ophthalmol. 1991;109:946-50.

29. Morlet N, Graham GG, Gatus B, et al. Pharmacokinetics of ciprofloxacin in the human eye: a clinical study and population pharmacokinetic analysis. Antimicrob Agents Chemother. 2000; 44:1674-9.

30. Brockhaus L, Goldblum D, Eggenschwiler L et al. Revisiting systemic treatment of bacterial endophthalmitis: a review of intravitreal penetration of systemic antibiotics. Clin Microbiol Infect. 2019. https://doi.org/10.1016/j.cmi.2019.01.017. [Epub ahead of print] 